\title{
Surrogate-Assisted Evolutionary Multitasking for Expensive Minimax Optimization in Multiple Scenarios
}

\author{
Handing Wang, School of Artificial Intelligence, Xidian University, Xi'an, China \\ Liang Feng, College of Computer Science, Chongqing University, Chongqing, China \\ Yaochu Jin, Department of Computer Science, University of Surrey, Guildford, UK \\ John Doherty, Department of Mechanical Engineering Sciences, University of Surrey, Guildford, UK
}

\begin{abstract}
Minimax optimization is a widely-used formulation for robust design in multiple operating or environmental scenarios, where the worst-case performance among multiple scenarios is the optimization objective requiring a large number of quality assessments. Consequently, minimax optimization using evolutionary algorithms becomes prohibitive when each quality assessment involves computationally expensive numerical simulations or costly physical experiments. This work employs evolutionary multitasking optimization and surrogate techniques to address the challenges of the high-dimensional search space and high computation cost. To this end, finding the worst-case scenario for different candidate solutions is considered as the optimization of multiple problems that can be solved simultaneously using the evolutionary multi-tasking optimization approach. In order to further speed up the proposed algorithm, a surrogate model in the joint space of the decision and scenario spaces is built to replace part of the expensive function evaluations. A generation-based model management strategy using a statistical hypothesis test is designed to manage the surrogate model. Experimental results on both benchmark problems and an airfoil design application indicate that the proposed algorithm can find satisfactory solutions with a very limited computational budget.
\end{abstract}

Index Terms-Surrogate, multitasking evolutionary algorithm, minimax optimization problem, robust optimization, multiscenario optimization.

\section{INTRODUCTION}

Robustness is of particular importance in engineering design when the system to be designed must operate in multiple scenarios [1], [2]. An ideal design is expected to achieve the best performance in all possible scenarios, which is often impossible for real-world applications due to the trade-off among those scenarios [3]. In other words, an optimal design in one single scenario might not have good performance in others [4], making the formulation of optimizing the performance in a single fixed scenario impractical [5]. Optimizing the performance in the worst-case scenario is one popular formulation of robust optimization to deal with multiple scenarios [6], which is known as minimax optimization [7]. In addition to the decision space of general optimization problems, a scenario space also needs to be coped with in minimax optimization problems. Minimax optimization can usually be achieved in a hierarchical way, where evaluating each solution in the design space requires an optimization search in the whole

Corresponding Author: Yaochu Jin (e-mail: yaochu.jin@surrey.ac.uk) scenario space. Traditional optimization algorithms such as mathematical programming [8]-[11], approximation methods [12], and branch-and-bound algorithms [13]-[15] have been employed to solve minimax optimization problems. However, their performance is less than satisfactory on the problems with continuous scenario space or nonanalytic objective functions.

Over the past two decades, a number of evolutionary algorithms (EAs) have shown promising performance on minimax optimization problems [16]-[19]. A straightforward fitness formulation for minimax optimization is to assess the worst-case scenario performance, which however, requires a large number of function evaluations. As a result, a hierarchical search of the optimal solution for minimax optimization incurs high computational costs, particularly when each quality evaluation is time-consuming.

Most minimax EAs adopt a coevolutionary approach [20] with populations searching the design and scenario spaces separately [7], [21]-[23]. Such parallel rather than hierarchical search used by these coevolutionary algorithms can considerably reduce the function evaluations required to achieve an acceptable solution. However, they easily get stuck in an endless optimization cycle in finding the optimum in the decision and scenario spaces [24] when the symmetrical condition as defined in [25] is not met. In fact, asymmetrical problems are more commonly seen than symmetrical problems in the real world [19]. Therefore, several rank-based evaluation methods [24], [25] were proposed to tackle this weakness of minimax coevolutionary algorithms. As the endless optimization cycle is caused by the coevolutionary structure, a few algorithms employ the hierarchical structure to deal with asymmetrical problems, where the scenario space is either traversed [26] or randomly sampled [25] for each candidate solution. To further improve the efficiency of the hierarchical structure, a recent minimax differential evolution (MMDE) algorithm [19] uses a min heap to allocate computational costs in the scenario space for different solutions. However, it still needs 100,000 function evaluations for problems with only two decision variables.

Many real engineering optimization problems have mediumscale decision variables and are driven by time-consuming function evaluations with a high degree of complexity [27]. For example, a three-dimensional computational fluid dynamics (CFD) simulation for one given scenario [5] might take minutes to hours, making existing minimax EAs impractical. 
Surrogate-assisted evolutionary algorithms (SAEAs) [28], [29] have shown both effectiveness and efficiency in solving expensive optimization problems [30], in which the expensive fitness evaluations are replaced by the computationally cheap surrogate models [31]-[33]. Those surrogate models are built and updated using a small number of real function evaluations. So far, surrogate models like the Gaussian processes (GP) [34]-[36] and radial basis function (RBF) networks [37]-[39] have been employed in two minimax EAs [31], [40] to reduce the function evaluations for finding the worst-case scenario for each candidate solution. However, neither of them can solve expensive minimax optimization problems taking into account multiple scenarios. The SAEA in [40] deals with the uncertainty in the decision space rather than that in the scenario space. The SAEA in [31] assumes that the evaluation of a given solution for the worst-scenario performance is expensive but the evaluation for a given solution in a given scenario is cheap, so it builds a GP model to approximate the worst-scenario objective function.

Existing EAs for solving minimax optimization problems consider all the worst-case scenario search processes for different solutions in the design space as independent optimization processes, and they employ an optimizer to repeatedly search the worst-case scenarios of all the individuals in the population. In fact, similar solutions in the decision space might have similar worst-case scenarios, which means the worst-case scenario search processes can be parallelized. Multitasking optimization (MTO) techniques [41], [42] can simultaneously solve multiple optimization problems in one single run, which takes advantage of the positive knowledge transfer between optimization problems (i.e., the similarity of the population in the decision space) [43], [44]. Equipped with an implicit parallelism, MTO has shown high efficiency on multi-objective optimization problems [45]-[48], bi-level optimization problems [49], expensive optimization problems [50], symbolic regression problems [51], sparse reconstruction [52], beneficiation processes [46], and capacitated vehicle routing problems [53]. The methodology of MTO naturally fits minimax optimization problems, if we consider the worstcase scenario search for different solutions in the population as different tasks.

In this paper, we make use of both surrogate and MTO to solve expensive minimax optimization problems within a limited number of function evaluations. The proposed algorithm is based on a popular MTO algorithm, the multifactorial evolutionary algorithm (MFEA) [42], in which function evaluations are replaced by a surrogate model (an RBF model). We term the proposed algorithm surrogate-assisted minimax multifactorial evolutionary algorithm (SA-MM-MFEA). The main contributions of this paper are as follows.

- We formulate the minimax worst-case multi-scenario optimization as a multitasking optimization problem with infinite tasks because of the continuous scenario space. Then, the scalar fitness calculation process in the MFEA is employed to solve the problem efficiently. In the proposed algorithm, although scenario and decision variables share one population for variation operations, the selection criteria for candidate scenarios and decisions are based on the multifactorial scalar fitness and the estimated worst-case scenarios, respectively.

- One single RBF model is built in the scenario-decision joint space to reduce the required number of expensive function evaluations.

- Since no existing model management strategy is applicable to minimax optimization problems, a new generationbased model management strategy using a statistical hypothesis test is introduced. It controls the setting of the RBF network and the infilling samples at a certain frequency so that the accuracy of the RBF model in the promising area can be further improved.

The rest of the paper is organized as follows. Firstly, we describe the minimax optimization problem and discuss its challenges in Section II. Section III briefly describes MTO techniques. Section IV details the proposed algorithm, including its framework, selection for both scenarios and solutions, and the model management strategy. To assess the performance of the proposed algorithm, we test it on existing low-dimensional benchmark problems and newly proposed medium-scale problems in Section V, and an airfoil design optimization example in Section VI as well. Finally, Section VII concludes the paper.

\section{Minimax Approach to Multi-Scenario OPTIMIZATION}

During the robust design process [2], the sources of uncertainty may come from decision space [54], objective space [55], or scenario space [5]. This work aims to find optimal solutions which are robust against the uncertainty introduced by multiple scenarios, which is well known as multi-scenario optimization [4]. To describe the uncertainty of scenario, a scenario space $\mathbf{s}$ is defined in the mathematical formulation of multi-scenario optimization problems as below:

$$
\underset{\mathbf{x} \in \Omega, \mathbf{s} \in \pi}{\arg \max } M_{R}(f(\mathbf{x}, \mathbf{s}), \mathbf{s}),
$$

where $\mathbf{x}$ is a $d$-dimensional decision variable in its feasible region $\Omega, \mathbf{s}$ is a $c$-dimensional scenario variable in its uncertainty range $\pi$ (either continuous or discrete), $f(\mathbf{x}, \mathbf{s})$ is the objective function, and $M_{R}$ is a robustness metric considering the whole scenario space. The worst-case scenario performance is one widely used metric to measure robustness, thus a multi-scenario minimax optimization problem can be written in Equation (2),

$$
\min _{\mathbf{x} \in \Omega} \max _{\mathbf{s} \in \pi} f(\mathbf{x}, \mathbf{s}),
$$

where $\max _{\mathbf{s} \in \pi} f(\mathbf{x}, \mathbf{s})$ is the worst-scenario performance value of $\mathbf{x}$ over the whole scenario space $\pi$. We term the optimal solution with its worst-case scenario $\left(\mathbf{x}^{*}, \mathrm{~s}^{*}\right)$.

To illustrate the optimization of a minimax optimization problem, we take candidate solutions (denoted by black dots) in Fig. 1 as an example. Those four solutions distributed in the decision space result in four different maximization subproblems in the scenario space. Before solving the outer minimization problem in Equation (2), EAs should find the worstcase scenario for each maximization subproblem, which makes 


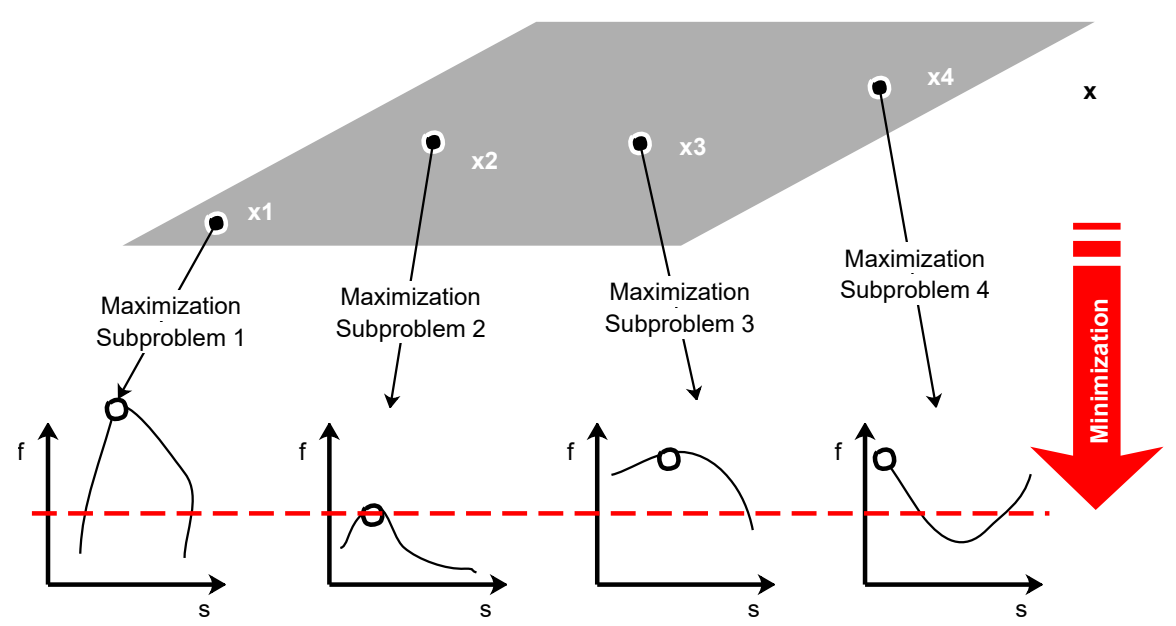

Fig. 1. Illustration of minimax optimization problems, where the upper level is the minimization problem in the decision space and the lower level is infinite maximization problems in the scenario space.

it a hierarchical optimization structure. Thus, the problem in Equation (2) can be decomposed into infinite maximization subproblems due to the continuous decision space $\Omega$, which brings prohibitive computation costs.

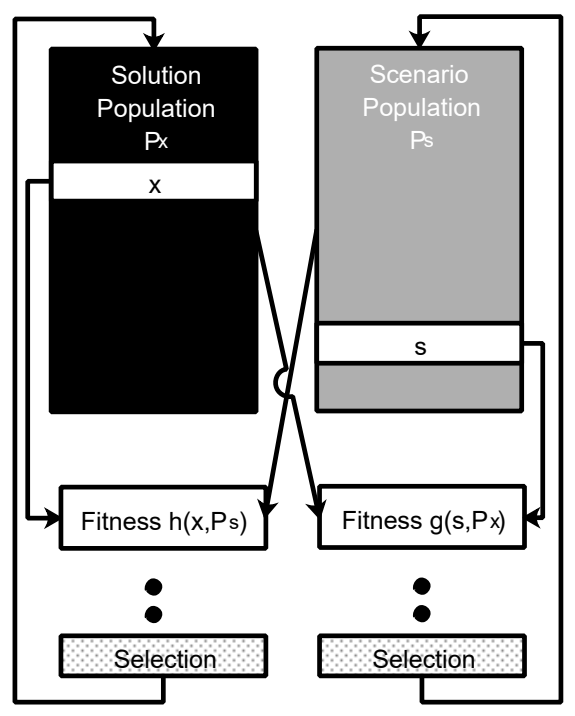

Fig. 2. Parallel optimization structure in minimax coevolutionary algorithm. The fitness functions of $P_{x}$ and $P_{s}$ for the solution and scenario space depend on each other.

One challenge for EAs to solve minimax optimization problems is to balance the search in the scenario and decision spaces. If too much effort is allocated to the worst-case scenario search, the search process in the decision space would be limited. If too little effort is allocated to the worst-case scenario search, the worst-case scenario objective value is not accurate, thereby misguiding the search direction in the decision space. This challenge becomes severe for minimax coevolutionary algorithms because of its parallel optimization structure for both spaces. The parallel structure [7] is shown in Fig. 2, where two populations $P_{x}$ and $P_{s}$ for the solution and scenario space are employed. For each population, a fitness function that is related to the other population is assigned. For the solution population $P_{x}$, the fitness $h\left(\mathbf{x}, P_{s}\right)$ (in Equation (3)) of a solution $\mathbf{x}$ is its worst objective value among the scenarios in $P_{s}$, which is given by:

$$
h\left(\mathbf{x}, P_{s}\right)=\max _{\mathbf{s} \in P_{s}} f(\mathbf{x}, \mathbf{s}) .
$$

For the scenario population $P_{s}$, the fitness $g\left(\mathbf{s}, P_{x}\right)$ (shown in Equation (4)) of a scenario $s$ is its best objective value among the solutions in $P_{x}$.

$$
g\left(\mathbf{s}, P_{x}\right)=\min _{\mathbf{x} \in P_{x}} f(\mathbf{x}, \mathbf{s}) .
$$

Based on those two fitness functions, two separate selection operations are applied to both populations. Therefore, the search in the decision and scenario spaces can be carried out in parallel in minimax coevolutionary algorithms. Such a parallel structure reduces the original search space in Fig. 1, where the worst-scenario search for $\mathrm{x}$ is restrained to $P_{s}$ rather than the whole scenario space. The reduced search is similar to separating optimization in the solution space and scenario space, which is conflicting for asymmetrical problems as $f\left(\mathbf{x}^{*}, \mathbf{s}\right) \leq f\left(\mathbf{x}^{*}, \mathbf{s}^{*}\right) \leq f\left(\mathbf{x}, \mathbf{s}^{*}\right)$ is not satisfied. Therefore, minimax coevolutionary algorithms cannot find the global optimum [19]. In addition, minimax optimization problems become even more challenging for EAs when a single calculation of $f(\mathbf{x}, \mathbf{s})$ is expensive.

\section{Preliminaries of Multitasking Optimization}

Conventional EAs serially solve different problems using a single population. As discussed in the previous section, a minimax optimization problem can be decomposed into an infinite number of maximization subproblems, making EAs inefficient for those massive subproblems. However, evolutionary multitasking optimization is an exception as it can simultaneously solve $k$ tasks (optimization problems $\left.\left\{\mathbf{x}_{1}, \ldots, \mathbf{x}_{k}\right\}=\arg \min \left\{f_{1}(\mathbf{x}), \ldots, f_{k}(\mathbf{x})\right\}\right)$ using a unified population [42]. 
MFEA [42] is a representative evolutionary multitasking optimization framework. Inspired by multifactorial inheritance [56], MFEA makes use of the diversity in the population to solve multiple problems by involving factorial rank $r$, skill factor $\tau$, and scalar fitness $\varphi$. Firstly, MFEA represents the decision variables of different tasks in a unified population and evaluates the solutions on all the tasks. Each solution will then be assigned a skill factor $\tau$ to label its best performing task. To be specific, if $r_{j}$ is the rank of a solution in the population on the $j$-th task, then it is associated with the task $\tau=\arg \min \left(r_{j}\right)$ with the best factorial rank among $k$ tasks, and its scalar fitness $\varphi$ is assigned as $1 / r_{\tau}$. Then, during the optimization process, each solution will only be evaluated on the task of its skill factor.

The main steps of MFEA are similar to conventional EAs, but the details of variation and evaluation are changed for multitasking as below:

- Initialization: MFEA generates an initial population $P$, evaluates $P$ on all the tasks, and calculates their skill factors $\tau$ and scalar fitness $\varphi$.

- Stopping condition: If the stopping condition is satisfied, MFEA outputs the obtained optimum of $k$ tasks; otherwise, it remains in the loop of variation, evaluation and selection.

- Variation: MFEA generates an offspring population $O$ via assortative mating, where crossover is applied to those two parents if two parents have a same skill factor or it is at a pre-defined probability; otherwise, mutation is applied to those two parents.

- Evaluation: Calculating one scalar fitness $\varphi$ requires $k$ function evaluations. When $k$ is a large number, the fitness calculation would be computationally demanding. Therefore, MFEA assigns $O$ skill factors via selective imitation, which depends on the skill factors of their parents. Then, only the assigned tasks of $O$ are evaluated, while the objective values of other unevaluated tasks are set as a very large number.

- Selection: MFEA re-calculates the scalar fitness of $P \cup O$ and selects half individuals with the best scalar fitness as the parent population for the next generation. Then, MFEA goes to the step "Stopping condition".

As mentioned in Section II, the difficulty of minimax optimization problems is the prohibitive computation costs spent on infinite maximization subproblems. The optimization processes of those similar maximization subproblems in a population share some mutual knowledge, which can be formulated as a multitasking optimization problem. Therefore, we believe that the implicit parallelism of the population and positive knowledge transfer of MFEA using the multifactorial scalar fitness can improve the effectiveness and efficiency of the evolutionary search on minimax optimization problems.

\section{Proposed Algorithm}

In this work, we borrow the implicit parallelism of the multifactorial scalar fitness in MFEA and the cheap cost of the RBF network to the proposed minimax optimization algorithm (SA-MM-MFEA) to address the issue of a limited computational budget for expensive minimax optimization problems.

\section{A. Framework}

SA-MM-MFEA follows the process of generic SAEAs, which includes model building, evolutionary operations (population initialization, variation, and selection), and model management [30]. The diagram of the proposed algorithm is illustrated in Fig. 3. At the very beginning of SA-MM-MFEA for solving a minimax optimization problem in Equation (2), a number of training data in the joint decision and scenario space $(\Omega \times \pi)$ are sampled using Latin hypercube sampling (LHS) to build an initial RBF network. Then, a population $P$ with $N$ random scenarios and solutions is initialized, where an individual is encoded as $(\mathbf{s}, \mathbf{x})$. Before the computational budget (a fixed number of expensive function evaluations) is exhausted, the proposed algorithm repeats the evolutionary search loop of variation (simulated binary crossover and polynomial mutation), evaluation, selection, and model management, where the evaluation, selection, and model management are different from the existing SAEAs.

All the scenario-solution pairs $(\mathbf{s}, \mathbf{x})$ in the population are estimated for the selection operations of scenario and decision variables. The selection operation in the scenario space is based on the multifactorial evaluations from MFEA since the worst-case scenario search for all the solutions in the population is viewed as a multitasking optimization problem. Then, the selection operation in the decision space is based on the estimated worst-case scenario performance. However, evaluating scenario-solution pairs are too expensive to conduct the search. Therefore, we employ the surrogate assistance using an RBF network that is built in $\Omega \times \pi$ and a generationbased model management strategy for minimax optimization problems to adaptively update the RBF network. The selection operations and surrogate assistance, two main contributions of this work (highlighted in Fig. 3), are explained in the following two sub-sections.

\section{B. Separate Selection Operations in Scenario and Decision Space}

Due to the hierarchical structure of minimax optimization problems, the selection operations in the scenario and decision spaces of SA-MM-MFEA are hierarchical. For the selection operation in the scenario space, the worst-case scenarios for the candidate solutions $\mathrm{x}^{i}$ are found. Unlike the existing minimax EAs that serially search the worst-case scenarios of the candidate $\mathbf{x}$ in the population, SA-MM-MFEA formulates such search process as a multitasking optimization problem for a parallel search. Then, based on those found worst-case scenarios, the worst-case scenario performance of each $\mathrm{x}^{i}$ can be estimated for the selection operation in the decision space. Both selection operations are shown in Fig. 4, where $N$ candidate solutions and scenarios are selected from a population with $2 N$ individuals.

The selection operation in the scenario space is based on a scalar fitness function borrowed from MFEA since the search of the worst-case scenarios of all $|P|$ solutions is parallelized 


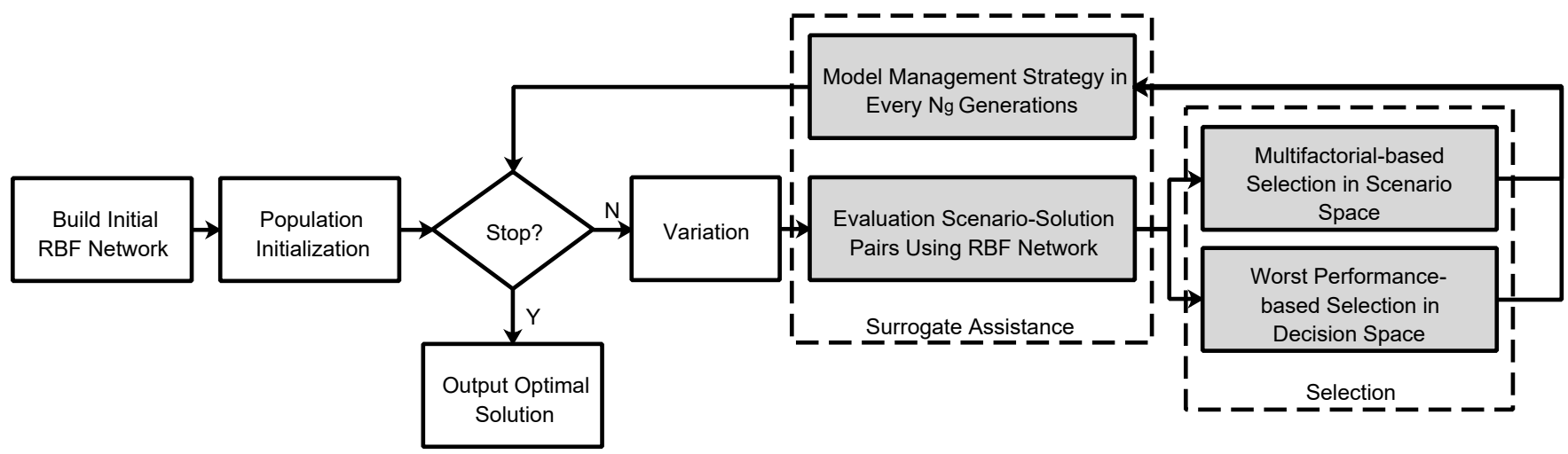

Fig. 3. A diagram of surrogate-assisted minimax multifactorial evolutionary algorithm

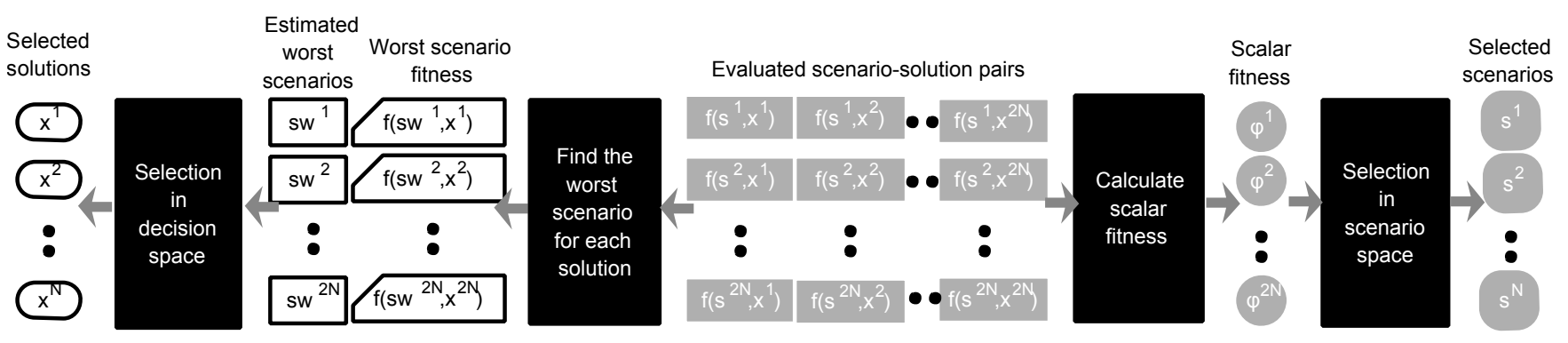

Fig. 4. Flow chart of the selection operations in the scenario and decision space, where the candidate scenarios are selected based on their scalar fitness and the candidate solutions are selected based on their estimated worst-case scenario performance.

by formulating as the following multitasking optimization problem:

$$
\underset{1 \leq j \leq|P|}{\mathbf{s}^{j}}=\underset{\mathbf{s} \in \pi}{\arg \max } f\left(\mathbf{x}^{j}, \mathbf{s}\right),
$$

where the $j$-th task is to find the worst-case scenario for the $j$-th candidate decision $\mathrm{x}^{j}$ in the population $P$. To calculate the scalar fitness $\varphi$ of each candidate scenario, each scenariosolution pair $\left(\mathbf{x}^{j}, \mathbf{s}^{i}\right)$ in $P$ is evaluated according to its performance $f\left(\mathbf{x}^{j}, \mathbf{s}^{i}\right)$ as shown in Fig. 4. The scalar fitness $\varphi^{i}$ of $\mathbf{s}^{i}$ can be calculated as below:

$$
\begin{aligned}
& \varphi^{i}=1 / \min R_{d}\left(\mathbf{s}^{i}, \varsigma^{j}\right), 1 \leq j \leq|P|, \\
& \varsigma^{j}=\left\{f\left(\mathbf{x}^{j}, \mathbf{s}^{1}\right), \ldots, f\left(\mathbf{x}^{j}, \mathbf{s}^{|P|}\right)\right\},
\end{aligned}
$$

where $\varsigma^{j}$ is the set of the objective values of $\mathrm{x}^{j}$ in all the candidate scenarios and $R_{d}\left(\mathbf{s}^{i}, \varsigma^{j}\right)$ is the rank function of $\mathbf{s}^{i}$ in $\varsigma^{j}$ in a descending order. Using Equation (6), the scalar fitness $\varphi^{i}$ represents the worst performance of $\mathbf{s}^{i}$ on those $|P|$ tasks in a comparative way. After sorting the individuals according to their scalar fitness in a descending order, the top $N$ scenarios are selected for the next generation.

The solutions should be selected based on their worstcase scenario performance. However, it is computationally prohibitive to precisely find the worst-case scenarios $\mathbf{s}^{*}$ for all the candidate solutions $\mathrm{x}^{i}$. Therefore, the proposed algorithm estimates their worst-case scenarios sw according to the scenario-solution pairs in $P$ which have been evaluated for calculating the scalar fitness $\varphi$. The estimated worst-case scenario $\mathbf{s w}^{j}$ for $\mathbf{x}^{j}$ can be written as the following equation:

$$
\mathbf{s w}^{j}=\underset{\mathbf{s}^{i}}{\arg \max } f\left(\mathbf{x}^{j}, \mathbf{s}^{i}\right),
$$

where $i$ is larger than 1 and smaller than $|P|$. Then, the fitness of $\mathbf{x}^{j}$ is set as $f\left(\mathbf{x}^{j}, \mathbf{s w}^{j}\right)$, which is an estimated worst-case scenario performance. SA-MM-MFEA sorts those fitness in an ascending order and selects the first $N$ solutions as shown in Fig. 4.

As explained in [24], coevolutionary algorithms cannot guarantee to find the optimum, because its fitness functions in Equations (3) and (4) are calculated based on a reduced search space. The proposed algorithm uses the parallelism of the scalar fitness function to deal with multiple worstcase search of different solutions, which alleviates the side effect of coevolutionary algorithms. Therefore, the proposed algorithm can outperform coevolutionary algorithms. Also, such MTO methodology parallels the worst-case scenario search of candidate solutions.

The selective imitation in scalar fitness calculation of MEFA is not employed in the proposed algorithm. The reason is that the proposed algorithm and MFEA deal with different multitasking optimization problems. MFEA synchronously solves $k$ fixed tasks, but SA-MM-MFEA handles an infinite number of tasks due to the continuous scenario space. Since the proposed algorithm searches solutions in the decision space as well as the scenario space over generations, it deals with $|P|$ different maximization problems varying with $\mathbf{x}$ in each generation. 


\section{Surrogate Assistance}

It requires $|P|^{2}$ evaluations of $f(\mathbf{x}, \mathbf{s})$ in each generation of the proposed algorithm, which makes it computationally expensive. A very straightforward idea to reduce the high computational cost is to use a surrogate model to replace the real evaluation of $f(\mathbf{x}, \mathbf{s})$, which is highlighted in Fig. 4.

To solve a minimax optimization problem with $d$ decision variables and $c$ scenario variables in Equation (2), an RBF network is built in the scenario-decision joint space $(d+c$ dimensional) to approximate $f(\mathbf{x}, \mathbf{s})$. We set the initial RBF network with $d+c$ Gaussian radial basis functions, and $11(d+$ c) initial training data $D_{T}$ is obtained via LHS in $\Omega \times \pi$. To train the RBF network, $d+c$ centers of radial basis functions are determined by applying the k-means algorithm, the widths of radial basis functions are the largest distances between those $d+c$ centers, and the weights of radial basis functions are calculated using the pseudo-inverse method [57].

Then, all the scenario-solution pairs $(\mathbf{s}, \mathbf{x})$ in the population are evaluated using the trained RBF network. Although the computation cost of evaluations is reduced, the approximation error of the RBF network might mislead the optimization process. To reduce that side-effect, SA-MM-MFEA adopts a generation-based model management strategy, where $3(d+c)$ new training data from a local promising area is sampled every $N_{g}$ generations.

The new generation-based model management strategy controls both the settings of the RBF network and infilling samples for minimax optimization problems. The proposed strategy as shown in Fig. 5 adjusts the model every $N_{g}$ generations, depending on the search progress of the last $N_{g}$ generations. Here, we assign sufficient generations to guarantee the convergence. Thus after $N_{g}$ generations, the population converges to a small area around the current best solution $\left(\mathbf{s w}^{\text {best }}, \mathbf{x}^{\text {best }}\right)$ (denoted by black triangles), a $l$ hypercube with $\left(\mathbf{s w}^{\text {best }}, \mathbf{x}^{\text {best }}\right.$ ) as its center (denoted by gray square area) is a promising local area. To assess whether that $l$-hypercube region is promising and whether those $N_{g}$ generations make any progress, $3(d+c)$ new data (written as $D_{N}$ and represented by white triangles) in that area is sampled for expensive evaluations. The baseline of the performance assessment of the last $N_{g}$ generations is the initial training data $D_{T}$ (denoted by gray dots). Note that the data $D_{L}$ (denoted by squares) which are sampled in the last time of model controlling cannot be employed as the baseline, because $D_{L}$ is not statical over different times of model management. A t-test between the objective values of $D_{N}$ and $D_{T}$ analyzes the differences of their distribution. According to the $p$-value $P V$ of the t-test, three cases in Fig. 5 might happen.

- Case 1 (RBF is not performing well): When the trained RBF network guides the proposed algorithm to a wrong area, the new samples $D_{N}$ degenerate the performance compared with previous samples. Such a degeneration can be presented from the distribution differences between $D_{T}$ and $D_{N}$ : the average objective value of $D_{N}$ $\left(A_{N}\right)$ is worse than that of $D_{T}\left(A_{T}\right)$, or $P V$ is larger than the best previous $p$-value $P V_{\text {best }}$. As the proposed algorithm starts with an RBF network with a small

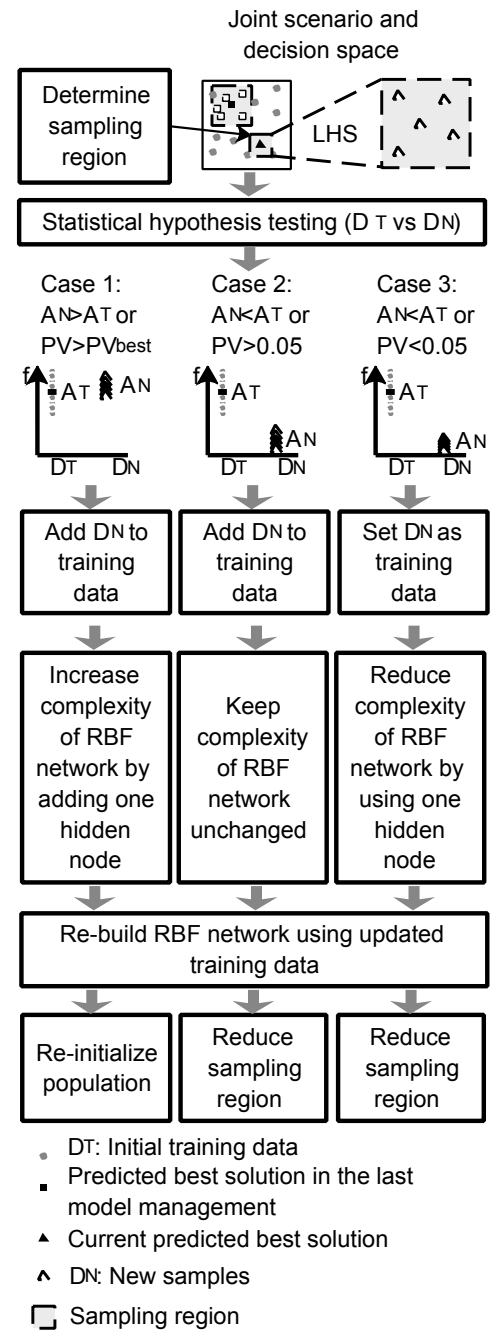

Fig. 5. Three cases in the new generation-based model management strategy.

number of hidden layer nodes, it is possible the current RBF network underfits. To improve the RBF network, the proposed algorithm increases the number of hidden nodes by one. In addition, the population has concentrated in a wrong area after $N_{g}$ generations, which needs a reinitialization to correct the search direction.

- Case 2 (RBF is performing well): The average objective value of $D_{N}\left(A_{N}\right)$ is better than that of $D_{T}\left(A_{T}\right)$ and $P V$ is larger than 0.05 , which shows the right search direction of the last $N_{g}$ generations but the objective value is not significantly improved. The proposed algorithm keeps the settings of the RBF network and the population for the next $N_{g}$ generations.

- Case 3 (RBF is performing well and a promising region is found): $D_{N}$ is sampled in a promising area which is predicted with best worst-scenario objective values, so it is very rare that $D_{N}$ is significantly better than $D_{T}$ which is randomly sampled in the whole solutionscenario joint space, i.e., the average objective value of $D_{N}\left(A_{N}\right)$ is better than that of $D_{T}\left(A_{T}\right)$ and $P V$ is smaller than 0.05 . In that case, the proposed algorithm 
has found a very promising region. To further exploit this promising local area, the proposed algorithm re-trains the RBF network with one hidden node using $D_{N}$.

The initial $11(d+c)$ training data are sampled using LHS, i.e., each dimension in the feasible space $\Omega \times \pi$ is divided into 10 grids, so we set the initial $l$ as $10 \%$ of the range of each dimension. When the model management strategy meets progressing cases ( 2 and 3 ), the population will further exploit the promising local area by reducing $l$ to $0.5 l$. After the resetting for the RBF network, population, and sampling region size, the RBF network is retrained using all the training data.

\section{EMPIRICAL STUDY}

In this section, we compare SA-MM-MFEA with the stateof-the-art minimax EAs on both low- and medium-dimensional benchmark problems. The following algorithms are compared:

- MMEA (minimax evolutionary algorithm) is an EA with the worst-case scenario performance as its fitness function. The worst-case scenario for each candidate solution is obtained via extra evolutionary search. Since MMEA does not use any mechanism for saving evaluations, it is the baseline algorithm.

- SA-MMEA (surrogate-assisted minimax evolutionary algorithm) replaces the real function evaluation with the $\mathrm{RBF}$ network in MMEA. In each generation, as most SAEAs [33], two solutions in the population are reevaluated to enrich the training data: the best solution and the most different solution compared with the training data.

- MMDE [19] is a differential evolution algorithm for minimax optimization problems, where a bottom-boosting scheme is employed to detect promising solutions and a partial-regeneration strategy is used to enhance the exploration ability.

- SA-MMDE (surrogate-assisted MMDE) replaces the real function evaluation with the RBF network. In each generation, two solutions in the population are re-evaluated to enrich the training data: the best solution and the most different solution compared with the training data. The comparison between MMDE and SA-MMDE can show the effect of surrogate models on MMDE.

- MM-MFEA (minimax multifactorial evolutionary algorithm) is an SA-MM-MFEA variant without surrogate models, which uses the real objective value to calculate the fitness and scalar fitness rather than the RBF network.

Among compared algorithms, only MMEA is an EA for minimax optimization in literature, and MMEA is a baseline algorithm. MMDE has shown its significant advantages over other minimax EAs in [19], so we only compared with MMDE in our experiment. To further analyze the contribution of surrogate models for solving expensive minimax problems, we also include the surrogate-assisted versions (SA-MMEA and SA-MMDE) of compared minimax EAs and non-surrogateassisted variant of the proposed algorithm as additional compared algorithms. In SA-MMEA and SA-MMDE, an RBF network with the same settings as that of the proposed algorithm is used to replace the real function evaluations, and both the best and most different solutions are added to the training data in the each generation.

In the comparative experiment, we apply the following settings. All the compared algorithms stop at the same number of real function evaluations. The population size of the compared SAEAs is 100 . However, due to the limited computational budget, the population size of non-surrogate-assisted algorithms is 10 . Specially, the population for the worst-case scenario search in MMEA is 2, and two parameters $K_{s}$ and $T$ controlling the number of real function evaluations in the bottom-boosting scheme and partial-regeneration strategy of MMDE are reduced to 19 and 1, whose original settings are 190 and 10 for inexpensive problems in [19]. $N_{g}$ in the proposed algorithm is set as 100. All the compared EAs use simulated binary crossover with $\eta=15$ and polynomial mutation with $\eta=15$, and all the compared DE use binomial recombination with $\mathrm{Cr}=0.5$ and mutation with $F=0.7$, which follows the settings of MMDE. The number of hidden nodes of the initial RBF network is set as $d+c$.

\section{A. Experiment on Low-Dimensional Problems}

Table I lists 6 widely-used minimax test problems with known optimum recommended in [19]. Since their dimensions of the decision and scenario spaces are up to 2, the stopping criterion of all the compared algorithms is set as 50 real function evaluations. Although the decision and scenario spaces are very low-dimensional, the dimension of actual search space is not low because of the hierarchical structure of minimax optimization problems. We run six compared algorithms (MMEA, SA-MMEA, MMDE, SA-MMDE, MMMFEA, and SA-MM-MFEA) on F1-6 for 30 independent times. The mean square error (MSE) of the obtained solutions to the true optimum are presented in Table II, where the results are analyzed by the Friedman test with the Bergmann-Hommel post-hoc test (SA-MM-MFEA is the control method and the significance level is 0.05) [58].

TABLE I

LOW-DIMENSIONAL TEST PROBLEMS.

\begin{tabular}{|c|c|c|}
\hline F1 & $\begin{array}{l}\text { Objective } \\
\text { Domain } \\
\text { Objective }\end{array}$ & $\begin{array}{l}f(x, s)=(x-5)^{2}-(s-5)^{2} \\
x \in[0,10], s \in[0,10] \\
\left(x^{*}, s^{*}\right)=(5,5)\end{array}$ \\
\hline $\mathrm{F} 2$ & $\begin{array}{l}\text { Objective } \\
\text { Domain } \\
\text { Objective }\end{array}$ & $\begin{array}{l}f(x, s)=\min \{3-0.2 x+0.3 s, 3+0.2 x-0.1 s\} \\
x \in[0,10], s \in[0,10] \\
\left(x^{*}, s^{*}\right)=(0,0)\end{array}$ \\
\hline F3 & $\begin{array}{l}\text { Objective } \\
\text { Domain } \\
\text { Objective }\end{array}$ & $\begin{array}{l}f(x, s)=\frac{\sin (x-s)}{\sqrt{x^{2}+s^{2}}} \\
x \in(0,10], s \in(0,10] \\
\left(x^{*}, s^{*}\right)=(10,2.125683)\end{array}$ \\
\hline $\mathrm{F} 4$ & $\begin{array}{l}\text { Objective } \\
\text { Domain } \\
\text { Objective }\end{array}$ & $\begin{array}{l}f(x, s)=\frac{\cos \sqrt{x^{2}+s^{2}}}{\sqrt{x^{2}+s^{2}}+10} \\
x \in[0,10], s \in[0,10] \\
\left(x^{*}, s^{*}\right)=(7.044146333751212,10 \text { or } 0)\end{array}$ \\
\hline F5 & $\begin{array}{l}\text { Objective } \\
\text { Domain } \\
\text { Objective }\end{array}$ & $\begin{array}{l}f(\mathbf{x}, \mathbf{s})=100\left(x_{2}-x_{1}^{2}\right)^{2}+\left(1-x_{1}\right)^{2} \\
\quad-s_{1}\left(x_{1}+x_{2}^{2}\right)-s_{2}\left(x_{1}^{2}+x_{2}\right) \\
\mathbf{x} \in[-0.5,0.5] \times[0,1], \mathbf{s} \in[0,10]^{2} \\
\left(\mathbf{x}^{*}, \mathbf{s}^{*}\right)=(0.5,0.25,0,0)\end{array}$ \\
\hline F6 & $\begin{array}{l}\text { Objective } \\
\text { Domain } \\
\text { Objective }\end{array}$ & $\begin{array}{l}f(\mathbf{x}, \mathbf{s})=100\left(x_{1}-2\right)^{2}+\left(x_{2}-2\right)^{2} \\
\quad+s_{1}\left(x_{1}^{2}-x_{2}\right)+s_{2}\left(x_{1}+x_{2}-2\right) \\
\mathbf{x} \in[-1,3]^{2}, \mathbf{s} \in[0,10]^{2} \\
\left(\mathbf{x}^{*}, \mathbf{s}^{*}\right)=(1,1, \text { any }, \text { any })\end{array}$ \\
\hline
\end{tabular}


TABLE II

MSE OF COMPARED ALGORITHMS ON F1-6. THE RESULTS ARE SHOWN IN THE FORM OF MEAN \pm STANDARD DEVIATION. THE RESULTS ARE ANALYZED BY THE FRIEDMAN TEST WITH THE BERGMANN-HOMMEL POST-HOC TEST (SA-MM-MFEA IS THE CONTROL METHOD AND THE SIGNIFICANCE LEVEL IS 0.05). THE BEST FITNESS VALUES AMONG ALL THE COMPARED ALGORITHMS FOR EACH PROBLEM ARE HIGHLIGHTED.

\begin{tabular}{|c|c|c|c|c|c|c|}
\hline & MMEA & SA-MMEA & MMDE & SA-MMDE & MM-MFEA & SA-MM-MFEA \\
\hline F1 & $25.4 \pm 8.7$ & $6.3 \pm 5.6$ & $15.1 \pm 12.1$ & $5.3 \pm 5.4$ & $25.3 \pm 9.0$ & $\mathbf{0 . 5} \pm \mathbf{0 . 6}$ \\
F2 & $57.6 \pm 49.1$ & $39.1 \pm 38.8$ & $35.1 \pm 24.6$ & $59.7 \pm 43.3$ & $57.5 \pm 45.8$ & $\mathbf{1 5 . 5} \pm \mathbf{3 3 . 9}$ \\
F3 & $63.0 \pm 34.8$ & $51.1 \pm 37.7$ & $50.4 \pm 46.4$ & $55.3 \pm 40.5$ & $69.4 \pm 51.3$ & $\mathbf{4 3 . 7} \pm \mathbf{3 0 . 7}$ \\
F4 & $21.8 \pm 20.5$ & $18.7 \pm 14.0$ & $11.0 \pm 13.1$ & $16.9 \pm 17.1$ & $15.0 \pm 15.9$ & $\mathbf{6 . 9} \pm \mathbf{1 0 . 9}$ \\
F5 & $43.1 \pm 25.0$ & $38.8 \pm 29.0$ & $29.8 \pm 24.2$ & $39.4 \pm 35.9$ & $40.4 \pm 32.2$ & $\mathbf{2 1 . 2} \pm \mathbf{3 7 . 0}$ \\
F6 & $95.0 \pm 50.3$ & $67.6 \pm 41.5$ & $45.2 \pm 36.5$ & $54.0 \pm 43.1$ & $48.6 \pm 36.0$ & $\mathbf{1 8 . 9} \pm \mathbf{2 8 . 8}$ \\
\hline Friedman rank & 5.7 & 3.7 & 2.3 & 4.0 & 4.3 & 1.0 \\
Adjusted $p$-value & $\mathbf{0 . 0 0}$ & $\mathbf{0 . 0 1}$ & 0.22 & $\mathbf{0 . 0 1}$ & $\mathbf{0 . 0 0}$ & NA \\
\hline
\end{tabular}

According to the Friedman rank, SA-MM-MFEA has the best performance, MMDE has the second best performance, and MMEA has the worst performance. It is not surprising that MMEA performs the worst, because it does not use any technique to save expensive evaluations. That is the reason why SA-MMEA using surrogate models to replace the real fitness evaluations outperforms MMEA. MMDE is a new well-performing minimax EA; it accelerates convergence by a parallel search in both decision and scenario spaces, making it much more efficient than MMEA. It is very interesting to note that SA-MMDE performs worse than MMDE. The approximation errors of the RBF network are inevitable even for low-dimensional problems, which may mislead the search direction of all surrogate-assisted versions of MMDE, MMEA, and MM-MFEA. In the experiment, SA-MMDE, SA-MMEA, and SA-MM-MFEA use the RBF network with similar accuracy, but the performance of SA-MMEA and SA-MM-MFEA improves compared to their original algorithms, while the performance of SA-MMDE deteriorates. This may be attributed to the different mechanisms they used, since SA-MMEA and SA-MM-MFEA search all the maximization subproblems in the population, but SA-MMDE only selects a few promising maximization subproblems in the search. In other words, the approximation errors of the RBF network may mislead one selection process in SA-MMEA and SA-MM-MFEA but two selection processes in SA-MMDE, making SA-MMDE more vulnerable to the approximation errors than the other two algorithms. The search space of low-dimensional problems is relatively small. As a result, the search performance of SA-MMDE is made worse rather than improved by the surrogate. Therefore, SA-MMDE is outperformed by MMDE. In addition, MFEA cannot outperform other compared algorithms except MMEA. In fact, MFEA cannot speed up the convergence on the multitasking optimization problems with a large number of tasks, because the calculation of the scalar fitness requires a large number of real fitness evaluations. However, the surrogate model assists MFEA to reduce the use of real fitness evaluations, making SA-MM-MFEA perform better than MMDE.

From the above results, we can make some observations. MMDE, surrogate models, and MFEA are helpful for solving expensive minimax optimization problems, but they result in different benefits. The bottom-boosting scheme in MMDE can efficiently identify the promising solutions, which is based on accurate fitness evaluations. Therefore, employing surrogate models in MMDE cannot further improve MMDE. MFEA can save expensive fitness evaluations for similar problems, but cannot solve minimax optimization problems with infinite tasks. Therefore, a simple combination of those techniques cannot lead to a satisfactory algorithm. The proposed algorithm uses a surrogate model to overcome the disadvantages of MFEA on minimax optimization problems and obtains the best performance within a very limited computational budget.

\section{B. Experiment on Median-Scale Problems}

It has been shown that many real-world engineering optimization problems have a median-scale decision space [5], [27]; thus the performance of minimax EAs on real-world problems cannot be represented by the results for lowdimensional test problems in the previous subsection. Unfortunately, there has not been any benchmark problems with higher dimensional decision variables for testing. To conduct the experiments in this sub-section, we construct a series of scalable test problems with the following general formula:

$$
\min _{\mathbf{x} \in \Omega} \max _{\mathbf{s} \in \pi}\left[f_{x s}\left(\mathbf{x}^{\prime}, \mathbf{s}^{\prime}\right)+f_{s}\left(\mathbf{s}^{\prime \prime}\right)+f_{x}\left(\mathbf{x}^{\prime \prime}\right)\right]
$$

where both the decision and scenario variables $\mathbf{x}$ and $\mathbf{s}$ can be divided into two groups $\mathbf{x}=\left\{\mathbf{x}^{\prime}, \mathbf{x}^{\prime \prime}\right\}$ and $\mathbf{s}=\left\{\mathbf{s}^{\prime}, \mathbf{s}^{\prime \prime}\right\}, f_{s}\left(\mathbf{s}^{\prime \prime}\right)$ and $f_{x}\left(\mathbf{x}^{\prime \prime}\right)$ are two functions only related to $\mathbf{s}^{\prime \prime}$ and $\mathbf{x}^{\prime \prime}$, and $f_{x s}\left(\mathbf{x}^{\prime}, \mathbf{s}^{\prime}\right)$ is a function related to both $\mathbf{x}^{\prime}$ and $\mathbf{s}^{\prime}$. Different combinations of those three functions result in five types of problems as shown in Table III, where type V is the hardest and type I (symmetrical) is the easiest. In this subsection, we employ existing benchmark problems (Ackley, Rastrigin, Rosenbrock, Griewank, and Ellipsoid functions [39]) as $f_{x s}$, $f_{s}$, and $f_{x}$ to build new test problems using Equation (8). Generally, the scenario space of real minimax optimization problems has lower dimensions than their decision space, so we set the dimensions of the scenario and decision spaces of the new problems as 2 and 28. In addition, either $\mathrm{x}$ or $\mathbf{s}$, which are two subfunctions, has two variable groups with equal dimensions. Taking type II problems as an example, the dimension numbers of $\mathbf{s}^{\prime}, \mathbf{s}^{\prime \prime}, \mathbf{x}^{\prime}$, and $\mathbf{x}^{\prime \prime}$ are $1,1,14$, and 14 , respectively, because neither $f_{x s}, f_{s}$, nor $f_{x}$ is zero. Thus, 20 new problems can be generated, and those problems are named with a type number and a constructor function number. For example, a test problem with the Ackley function as nonzero $f_{x s}, f_{s}$, and $f_{x}$ is named as II-Ackley. To evaluate the results, we employ MMEA using a large number of function 
evaluations to estimate the true optimum, where the population size is 100 , the population for the worst-case scenario search is 100 , and the stopping criterion is 100 generations.

TABLE III

FIVE TYPES OF NEW SCALABLE TEST PROBLEMS

\begin{tabular}{|c|c|c|c|}
\hline Type & $f_{x s}$ & $f_{s}$ & $f_{x}$ \\
\hline I & zero & nonzero & nonzero \\
II & nonzero & nonzero & nonzero \\
III & nonzero & zero & nonzero \\
IV & nonzero & nonzero & zero \\
V & nonzero & zero & zero \\
\hline
\end{tabular}

Compared with the test problems in the previous subsection, the new test problems are higher-dimensional than that in the previous subsection, so the stopping criterion is extended to 1000 real function evaluations. We run six compared algorithms (MMEA, SA-MMEA, MMDE, SA-MMDE, MMMFEA, and SA-MM-MFEA) on the new test problems for 30 independent times. The MSE of the obtained solutions to the true optimum are shown in Table IV, where the results are analyzed by the Friedman test with the Bergmann-Hommel post-hoc test (SA-MM-MFEA is the control method and the significance level is 0.05) [58].

Generally, SA-MM-MFEA performs the best on those median-scale problems, SA-MMEA and SA-MMDE perform worse than SA-MM-MFEA, and the other three compared algorithms have the worst performance. As the dimension of the decision space increases, the searching space dramatically increases, therefore the results in Table IV are different from that in Table II. Due to the large decision space of the test problems, non-surrogate-assisted EAs (MMEA, MMDE, and MM-MFEA) cannot outperform surrogate-assisted EAs (SA-MMEA, SA-MMDE, and SA-MM-MFEA). In particular, MMDE, which is the second best algorithm for lowdimensional problems, performs the second worst on those median-scale problems.

Type I problems have separable scenario and decision variables, so they are symmetrical problems. Although these problems are the easiest problems among the test problems in this sub-section, non-surrogate-assisted EAs (MMEA, MMDE, and MM-MFEA) cannot outperform surrogate-assisted EAs (SA-MMEA, SA-MMDE, and SA-MM-MFEA). MM-MFEA is the best performing non-surrogate-assisted EA on type I problems, and its surrogate version SA-MM-MFEA outperforms all the compared algorithms except for I-Rosenbrock. MMDE is better than MMEA, but it does not result in the better performance of SA-MMDE than SA-MMEA. The reason has been explained in the pervious section: MMDE is more sensitive to approximation errors than MMEA.

Type II problems have both separable and nonseparable scenario and decision variables, which makes them asymmetrical. SA-MM-MFEA has greater advantage than other algorithms on type II problems than type I problems, where it performs the best on all the five type II problems. Since type II problems are harder than type I problems, SA-MMDE degenerates its performance.

Type III problems have a larger number of nonseparable scenario variables than type II problems, and the type IV problems have a larger number of nonseparable decision variables than type II problems. The results of compared algorithms are similar to the results on type II problems, where SA-MM-MFEA has the best performance and MMEA has the worst performance. The only difference is that SA-MMEA obtained the best results on III-Rosenbrock. Type V problems have nonseparable scenario and decision variables, which is the hardest type among those five types. SA-MM-MFEA still outperforms other compared algorithms on all five type $\mathrm{V}$ problems.

According to the Friedman ranks in Table IV, the performance of compared algorithms changes slightly for different types of problems. When the computational budget is limited for solving medium-scale minimax optimization problems, the main challenge for minimax EAs is the large search space rather than their asymmetry. While general expensive problems with 30 decision and scenario variables are still challenging for EAs, expensive minimax optimization problems with 30 decision variables are even harder. With the help of surrogate model, the proposed algorithm significantly reduces the computation cost, enabling it to outperform MMDE. Furthermore, the MTO technique employed in the proposed algorithm enhances the search efficiency and leads the better performance than other surrogate-assisted minimax EAs. From the results in both subsections, we can conclude that the proposed algorithm can find the better solution than existing methods.

\section{Experiment on Model Management Strategy}

The model management strategy in SA-MM-MFEA is different from the existing strategies, because it is designed for expensive minimax problems. To illustrate the contribution of the statistical hypothesis test-based model management, we exclude the statistical hypothesis test-based control for RBF network and promising region size from the proposed model management strategy (i.e., LHS is adopted for new samples, but the sampling region size and the number of hidden layer nodes of the RBF network are fixed) and compare it with the original strategy on the type $\mathrm{V}$ problems. We run SAMM-MFEAs with and without statistical hypothesis test-based control for 30 independent times, and the results are presented in Table $\mathrm{V}$ and analyzed by the Wilcoxon signed-rank test (significance level=0.05) [58].

The results of SA-MM-MFEA(SHT) are significantly better than those of SA-MM-MFEA(NSHT) on all the type V problems, which indicates the effectiveness of the proposed model management strategy for minimax optimization problems. In the statistical hypothesis test-based model management strategy, the new data are sampled from an adaptive sampling region around the predicted best solution, and the number of hidden layer nodes of the RBF network changed over the optimization process, which enables SA-MM-MFEA to further update the RBF network in a small local area and to exploit the promising region.

To further study the contributions of different strategies in three cases for SA-MM-MFEA, we show the average percentage in performance improvement of those three cases in Fig. 6. It shows that the different strategies in three cases 
TABLE IV

MSE OF COMPARED ALGORITHMS ON NEW MEDIAN-SCALE BENCHMARK PROBLEMS. THE RESULTS ARE SHOWN IN THE FORM OF MEAN \pm STANDARD DEVIATION. THE RESUltS ARE ANALYZED By THE FRIEDMAN TEST WITH THE BERGMANN-HOMMEL POST-HOC TEST (SA-MM-MFEA IS THE CONTROL METHOD AND THE SIGNIFICANCE LEVEL IS 0.05). THE BEST FITNESS VALUES AMONG ALL THE COMPARED ALGORITHMS FOR EACH PROBLEM ARE HIGHLIGHTED.

\begin{tabular}{|c|c|c|c|c|c|c|}
\hline & MMEA & SA-MMEA & MMDE & SA-MMDE & MM-MFEA & SA-MM-MFEA \\
\hline I-Griewank & $5.6 \pm 1.0$ & $1.2 \pm 0.4$ & $4.9 \pm 1.2$ & $1.2 \pm 0.6$ & $5.1 \pm 0.7$ & $\mathbf{0 . 0} \pm \mathbf{0 . 0}$ \\
\hline I-Ackley & $7.9 \pm 1.6$ & $2.6 \pm 1.8$ & $6.9 \pm 1.9$ & $11.4 \pm 3.0$ & $6.0 \pm 1.2$ & $\mathbf{0 . 0} \pm \mathbf{0 . 0}$ \\
\hline I-Rastrigin & $6.6 \pm 1.3$ & $1.0 \pm 0.6$ & $7.1 \pm 1.5$ & $1.2 \pm 0.8$ & $6.2 \pm 1.2$ & $\mathbf{0 . 0} \pm \mathbf{0 . 0}$ \\
\hline I-Ellipsoid & $5.8 \pm 0.9$ & $1.0 \pm 0.4$ & $5.5 \pm 0.9$ & $0.8 \pm 0.4$ & $5.7 \pm 1.0$ & $\mathbf{0 . 0} \pm \mathbf{0 . 0}$ \\
\hline I-Rosenbrock & $9.4 \pm 2.3$ & $4.7 \pm 2.0$ & $10.4 \pm 1.3$ & $4.4 \pm 2.8$ & $8.4 \pm 2.1$ & $4.9 \pm 2.4$ \\
\hline Friedman rank & 5.4 & 2.4 & 4.8 & 2.8 & 4.2 & 1.4 \\
\hline Adjusted $p$-value & 0.00 & 0.40 & 0.00 & 0.24 & 0.02 & NA \\
\hline II-Griewank & $5.4 \pm 0.7$ & $1.3 \pm 0.4$ & $4.9 \pm 1.0$ & $1.6 \pm 1.0$ & $5.1 \pm 0.8$ & $\overline{0.0} \pm \mathbf{0 . 0}$ \\
\hline II-Ackley & $7.4 \pm 1.4$ & $4.0 \pm 1.0$ & $7.0 \pm 1.6$ & $13.3 \pm 1.9$ & $6.2 \pm 1.5$ & $0.1 \pm 0.0$ \\
\hline II-Rastrigin & $6.6 \pm 1.5$ & $0.9 \pm 0.5$ & $7.1 \pm 1.5$ & $1.1 \pm 0.5$ & $6.6 \pm 1.2$ & $\mathbf{0 . 0} \pm \mathbf{0 . 0}$ \\
\hline II-Ellipsoid & $5.9 \pm 1.1$ & $1.0 \pm 0.3$ & $5.4 \pm 1.1$ & $0.7 \pm 0.4$ & $5.4 \pm 0.8$ & $\mathbf{0 . 0} \pm \mathbf{0 . 0}$ \\
\hline II-Rosenbrock & $9.7 \pm 1.7$ & $6.6 \pm 2.1$ & $12.0 \pm 2.0$ & $5.9 \pm 1.8$ & $9.5 \pm 2.6$ & $5.0 \pm 2.0$ \\
\hline Friedman rank & 5.4 & 2.4 & 4.8 & 3.2 & 4.2 & 1.0 \\
\hline Adjusted $p$-value & 0.00 & 0.24 & 0.00 & 0.06 & 0.01 & NA \\
\hline III-Griewank & $5.7 \pm 1.1$ & $1.3 \pm 0.4$ & $4.8 \pm 0.8$ & $1.4 \pm 0.7$ & $5.1 \pm 0.8$ & $\mathbf{0 . 0} \pm \mathbf{0 . 0}$ \\
\hline III-Ackley & $6.2 \pm 1.1$ & $0.2 \pm 0.1$ & $7.0 \pm 1.4$ & $0.4 \pm 0.3$ & $5.9 \pm 1.2$ & $\mathbf{0 . 0} \pm \mathbf{0 . 0}$ \\
\hline III-Rastrigin & $6.4 \pm 1.5$ & $0.8 \pm 0.4$ & $7.7 \pm 1.4$ & $1.3 \pm 0.9$ & $6.1 \pm 0.9$ & $\mathbf{0 . 0} \pm \mathbf{0 . 0}$ \\
\hline III-Ellipsoid & $5.8 \pm 0.6$ & $1.1 \pm 0.4$ & $5.1 \pm 1.1$ & $0.8 \pm 0.4$ & $5.5 \pm 0.8$ & $\mathbf{0 . 0} \pm \mathbf{0 . 0}$ \\
\hline III-Rosenbrock & $10.0 \pm 2.6$ & $5.5 \pm 2.1$ & $11.1 \pm 1.4$ & $6.5 \pm 2.8$ & $8.8 \pm 2.2$ & $6.8 \pm 2.5$ \\
\hline Friedman rank & 5.4 & 2.0 & 5.2 & 2.6 & 4.4 & 1.4 \\
\hline Adjusted $p$-value & 0.00 & 0.61 & 0.00 & 0.31 & 0.01 & NA \\
\hline IV-Griewank & $5.8 \pm 1.0$ & $1.2 \pm 0.5$ & $4.8 \pm 0.8$ & $1.3 \pm 1.0$ & $5.0 \pm 0.6$ & $\mathbf{0 . 0} \pm \mathbf{0 . 0}$ \\
\hline IV-Ackley & $7.1 \pm 0.9$ & $4.4 \pm 1.1$ & $7.6 \pm 2.1$ & $14.0 \pm 2.4$ & $6.1 \pm 1.3$ & $0.4 \pm 0.7$ \\
\hline IV-Rastrigin & $6.9 \pm 1.0$ & $0.8 \pm 0.5$ & $7.3 \pm 1.6$ & $1.4 \pm 0.8$ & $6.5 \pm 1.0$ & $\mathbf{0 . 0} \pm \mathbf{0 . 0}$ \\
\hline IV-Ellipsoid & $5.9 \pm 1.2$ & $1.0 \pm 0.4$ & $5.3 \pm 0.9$ & $0.8 \pm 0.5$ & $5.5 \pm 0.8$ & $\mathbf{0 . 0} \pm \mathbf{0 . 0}$ \\
\hline IV-Rosenbrock & $8.6 \pm 2.0$ & $4.5 \pm 2.2$ & $11.1 \pm 1.7$ & $3.9 \pm 1.9$ & $8.5 \pm 2.8$ & $3.5 \pm 2.0$ \\
\hline Friedman rank & 5.2 & 2.4 & 5.0 & 3.2 & 4.2 & 1.0 \\
\hline Adjusted $p$-value & 0.00 & 0.24 & 0.00 & 0.06 & 0.00 & NA \\
\hline V-Griewank & $5.5 \pm 0.9$ & $1.3 \pm 0.4$ & $5.0 \pm 1.0$ & $1.3 \pm 0.9$ & $5.3 \pm 0.9$ & $\mathbf{0 . 0} \pm \mathbf{0 . 0}$ \\
\hline V-Ackley & $6.7 \pm 1.2$ & $0.0 \pm 0.0$ & $6.6 \pm 1.5$ & $0.1 \pm 0.1$ & $5.7 \pm 1.5$ & $\mathbf{0 . 0} \pm \mathbf{0 . 1}$ \\
\hline V-Rastrigin & $6.6 \pm 1.3$ & $1.0 \pm 0.6$ & $7.2 \pm 1.5$ & $1.3 \pm 0.6$ & $6.4 \pm 1.1$ & $\mathbf{0 . 0} \pm \mathbf{0 . 0}$ \\
\hline V-Ellipsoid & $5.9 \pm 0.9$ & $0.9 \pm 0.3$ & $5.3 \pm 0.8$ & $0.9 \pm 0.5$ & $5.5 \pm 0.9$ & $\mathbf{0 . 0} \pm \mathbf{0 . 0}$ \\
\hline V-Rosenbrock & $9.8 \pm 2.3$ & $5.2 \pm 2.0$ & $10.7 \pm 1.7$ & $5.8 \pm 2.5$ & $9.0 \pm 2.5$ & $4.4 \pm 2.4$ \\
\hline Friedman rank & 5.6 & 2.6 & 5.0 & 2.4 & 4.4 & 1.0 \\
\hline Adjusted $p$-value & 0.00 & 0.18 & 0.00 & 0.24 & 0.00 & NA \\
\hline
\end{tabular}

TABLE V

MSE OF SA-MM-MFEAS WITH AND WITHOUT THE STATISTICAL HYPOTHESIS TEST-BASED CONTROL (SHORT FOR SA-MM-MFEA(SHT) AND SA-MM-MFEA(NSHT)) ON THE TYPE V PROBLEMS. THE RESULTS ARE SHOWN IN THE FORM OF MEAN \pm STANDARD DEVIATION. THE RESULTS ARE ANALYZED BY THE WILCOXON SIGNED-RANK TEST (SIGNIFICANCE LEVEL $=0.05$ ). THE SIGNIFICANT ALGORITHMS FOR EACH PROBLEM ARE HIGHLIGHTED IN BOLD FACE.

\begin{tabular}{|c|c|c|}
\hline & SA-MM-MFEA(SHT) & SA-MM-MFEA(NSHT) \\
\hline V-Griewank & $\mathbf{0 . 0} \pm \mathbf{0 . 0}$ & $0.5 \pm 0.1$ \\
V-Ackley & $\mathbf{0 . 0} \pm \mathbf{0 . 1}$ & $0.3 \pm 0.2$ \\
V-Rastrigin & $\mathbf{0 . 0} \pm \mathbf{0 . 0}$ & $0.7 \pm 0.2$ \\
V-Ellipsoid & $\mathbf{0 . 0} \pm \mathbf{0 . 0}$ & $0.6 \pm 0.2$ \\
V-Rosenbrock & $\mathbf{4 . 4} \pm \mathbf{2 . 4}$ & $6.6 \pm 2.7$ \\
\hline
\end{tabular}

effectively improve the performance, but their percentages in performance improvement vary on different problems. Especially, Case 1 never appeared in the search of the uni-modal problem V-Ellipsoid, and the proposed model management strategy can quickly exploit the area of the optimum, which is shown by the high percentage in performance improvement in Case 3. Therefore, the proposed model management strategy adaptively adjusts the RBF network according to the problem landscape.

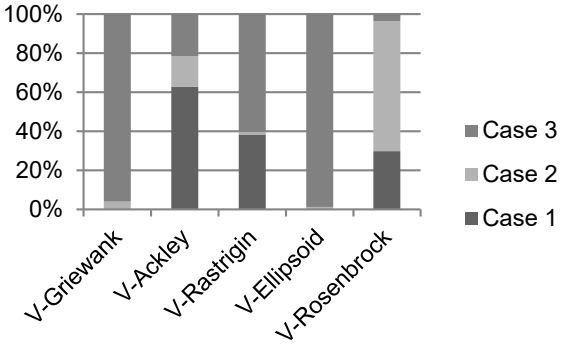

Fig. 6. Average percentage in performance improvement of Cases 1, 2, and 3 in SA-MM-MFEA on the type $\mathrm{V}$ problems.

\section{Sensitivity Analysis of the Population Size of Non- Surrogate-Assisted Algorithms}

In the comparative experiments on expensive minimax optimization problems, the stopping criterion is a fixed small number of real function evaluations. To ensure a number of generations of non-surrogate-assisted algorithms (MMEA, MMDE, and MM-MFEA), we set their populations in a small size, which is different from their settings on inexpensive problems. In this subsection, we employ II-Rastrigin as a test problem and 1000 real function evaluations as the computational budget to discuss the effect of large population sizes on 
non-surrogate-assisted algorithms.

MMEA adopts two populations: one is the population with $N$ individuals for the optimal solution search, and the other one the population with $N_{s w}$ individuals for the worst-case scenario search. We run MMEA with different $N$ and $N_{s w}$ on II-Rastrigin for 30 independent times, and the results are shown in Table VI. As $N$ decreases, the number of generations increases, and the performance of MMEA improves. Similarly, a small $N_{s w}$ leads to a better performance of MMEA than a large $N_{s w}$, because a small population allows for a larger number of generations for the worst-case scenario search.

TABLE VI

MSE OF MMEA WITH DIFFERENT $N$ AND $N_{s w}$ ON II-RASTRIGIN. THE SMALLEST MSE IS HIGHLIGHTED IN BOLD FACE.

\begin{tabular}{|c|c|c|c|c|}
\hline & $N=100$ & $N=50$ & $N=20$ & $N=10$ \\
\hline$N_{s w}=2$ & $7.7 \pm 1.4$ & $7.9 \pm 1.1$ & $7.3 \pm 0.8$ & $\mathbf{6 . 6} \pm \mathbf{1 . 1}$ \\
$N_{s w}=10$ & $7.8 \pm 1.2$ & $7.9 \pm 1.0$ & $7.7 \pm 1.2$ & $7.0 \pm 1.3$ \\
\hline
\end{tabular}

MMDE has two parameters $\left(K_{s}\right.$ and $\left.T\right)$ to control its population size. We test MMDE with different $K_{s}$ and $T$ on II-Rastrigin for 30 independent times, and the results are shown in Table VII. As $K_{s}$ and $T$ increase, the performance of MMDE degenerates due to insufficient generations.

TABLE VII

MSE OF MMDE WITH DIFFERENT $K_{S}$ AND $T$ ON II-RASTRIGIN. THE SMALLEST MSE IS HIGHLIGHTED IN BOLD FACE.

\begin{tabular}{|c|c|}
\hline$K_{s}=19, T=1$ & $\mathbf{7 . 1} \pm \mathbf{1 . 5}$ \\
$K_{s}=38, T=2$ & $7.2 \pm 1.2$ \\
$K_{s}=95, T=5$ & $7.4 \pm 1.4$ \\
$K_{s}=190, T=10$ & $12.4 \pm 2.3$ \\
\hline
\end{tabular}

MM-MFEA is a variant of the proposed algorithm without surrogate assistance. We test MM-MFEA with different population sizes $N$ on II-Rastrigin for 30 independent times. As MM-MFEA evaluates $N^{2}$ scenario-solution pairs in each generation, the computational budget (1000 real function evaluations) limits the largest $N$ to 50. As the results in Table VIII indicate, MM-MFEA using $N=10$ finds better solutions than MM-MFEA using $N=20$ and $N=50$.

TABLE VIII

MSE OF MM-MFEA WITH DIFFERENT POPULATION SIZES $N$ ON II-RASTRIGIN. THE SMALLEST MSE IS HIGHLIGHTED IN BOLD FACE.

\begin{tabular}{|c|c|c|}
\hline$N=10$ & $N=20$ & $N=50$ \\
\hline $\mathbf{6 . 6} \pm \mathbf{1 . 2}$ & $6.7 \pm 1.0$ & $7.1 \pm 1.1$ \\
\hline
\end{tabular}

From the above experimental results, we can conclude that a small population size for non-surrogate-assisted algorithms can enhance their performance on expensive minimax optimization problems. The main reason is that a small population can increase the number of generations, which benefits the optimization process when the computational budget is limited.

\section{Airfoil Robust Design}

A robust airfoil should be well-performing in a variety of scenarios (i.e., different velocities and lifts) [5], [59], making the design process a minimax optimization process.
Additionally, evaluating airfoil performance in each scenario requires a CFD simulation that takes minutes. To validate the performance of the proposed algorithm on real-world applications, we use an RAE2822 airfoil test case in the GARTEUR AG52 project $^{1}$ in this section.

The scenario space of this RAE2822 minimax optimization problem is two-dimensional and consists of the Mach number $M$ (speed) and lift coefficient $C_{L}$. The objective is to minimize the worst-scenario drag over lift ratio:

$$
f_{\text {Airfoil }}=\max _{M \in \Upsilon, C_{L} \in \Gamma} \frac{C_{D w}\left(M, C_{L}\right)}{C_{L}},
$$

where $C_{D w}\left(M, C_{L}\right)$ is the drag coefficient in the scenario $\left(M, C_{L}\right)$ and calculated via an expensive CFD simulation. Additionally, the ranges of $M$ and $C_{L}$ are defined as $\Upsilon=$ $[0.704,0.734]$ and $\Gamma=[0.704,0.824]$ in Equation (9). The geometry of the airfoil is defined by a camber and fixed thickness distribution. Using six Henne-Hicks functions [60] in Equations (10-15), a camber shape of a design can be calculated as Equation (16),

$$
\begin{gathered}
f_{1}=\frac{x^{0.5}(1-x)}{e^{15 x}} \\
f_{2}=\sin \left(\pi x^{0.25}\right)^{3} \\
f_{3}=\sin \left(\pi x^{0.757}\right)^{3} \\
f_{4}=\sin \left(\pi x^{1.357}\right)^{3} \\
f_{5}=\frac{x^{0.5}(1-x)}{e^{10 x}} \\
f_{6}=\sin \left(\pi x^{4}\right) \\
y_{c}=y_{c}^{b}+\sum_{i=1}^{6} a_{i} f_{i},
\end{gathered}
$$

where $y_{c}^{b}$ is the camber shape of baseline airfoil and $a_{i}$ are weights of those six Henne-Hicks functions. Thus, six decision variables $\left(a_{1}, \ldots, a_{6}\right)$ determine the camber shapes and the airfoil shapes of candidate designs.

We compare MMEA, SA-MMEA, MMDE, SA-MMDE, MM-MFEA, and SA-MM-MFEA using 250 CFD simulations on the airfoil minimax optimization problem. All compared algorithms are run for 30 times independently. For each obtained solution, CFD simulations in $31 \times 13$ uniform scenarios are calculated in order to estimate its worst-case performance. The results are shown in Table IX. Consistent with the results on the benchmark problems, the proposed algorithm SAMM-MFEA outperforms other compared algorithms. In other words, with the assistance of multitasking optimization and surrogate techniques, SA-MM-MFEA can efficiently find an airfoil with low drag over lift ratio in multiple scenarios.

\footnotetext{
${ }^{1}$ http://www.garteur.org/
} 
TABLE IX

OPTIMAL SOLUTIONS OBTAINED BY MMEA, SA-MMEA, MMDE, SA-MMDE, MM-MFEA, AND SA-MM-MFEA ON THE RAE2822 AIRFOIL PROBLEM.

\begin{tabular}{|c|c|}
\hline Algorithm & Obtained solution \\
\hline MMEA & $0.0098 \pm 0.0105$ \\
SA-MMEA & $0.0123 \pm 0.0137$ \\
MMDE & $0.0055 \pm 0.0050$ \\
SA-MMDE & $0.0070 \pm 0.0033$ \\
MM-MFEA & $0.0077 \pm 0.0126$ \\
SA-MM-MFEA & $\mathbf{0 . 0 0 3 1} \pm \mathbf{0 . 0 0 2 4}$ \\
\hline
\end{tabular}

\section{CONCLUSIONS}

In this work, we proposed a surrogate-assisted multifactorial evolutionary algorithm to address the challenges of expensive minimax optimization problems for robust design. The proposed algorithm takes the advantage of the implicit parallelism from MFEA and from surrogate models to accelerate the search of the minimax EA. For the minimax optimization problems in particular, the model management strategy is redesigned using a statistical hypothesis test (t-test) to assess the optimization progress. According to the experimental results on both benchmark problems and airfoil design problems, the proposed algorithm adaptively updates the surrogate model and corrects the search direction. The experimental results on both low- and medium-dimensional problems indicate that the proposed algorithm can find satisfactory solutions using a very limited computational budget. It is worth noting that the proposed algorithm cannot be applied to the problems with a discrete scenario space because the joint decision and scenario space becomes mixed, which cannot be handled by the employed surrogate model. To solve the minimax problems with a discrete scenario space, a surrogate model for mixed variables needs to replace the RBF network with Gaussian radial basis functions in the proposed algorithm.

Although the proposed algorithm inherits the implicit parallelism of MFEA to efficiently search for optimal solutions for worst-case scenarios, it does not use the selective imitation because of the changing tasks over generations. The tasks in the population changes over generations, but there could be a part of promising tasks that can be kept for a number of generations. A new selective imitation should be able to assign both new and old tasks to individuals in the population to further accelerate the algorithm. In addition, the worst-case scenario search for a number of solutions can act as source problems, then a multi-source selective transfer framework [61] can be employed to find the worst-case scenario of other solutions via transfer learning rather than repeated similar search processes.

\section{ACKNOWLEDGEMENTS}

This work was supported in part by the National Natural Science Foundation of China (No. 61976165, 61876025 and 61590922).

\section{REFERENCES}

[1] R. Suri and K. Otto, "Manufacturing system robustness through integrated modeling," Journal of Mechanical Design, vol. 123, no. 4, pp. 630-636, 2001.
[2] H.-G. Beyer and B. Sendhoff, "Robust optimization-a comprehensive survey," Computer methods in applied mechanics and engineering, vol. 196, no. 33-34, pp. 3190-3218, 2007.

[3] L. Zhu, K. Deb, and S. Kulkarni, "Multi-scenario optimization using multi-criterion methods: A case study on byzantine agreement problem," in Evolutionary Computation (CEC), 2014 IEEE Congress on. IEEE, 2014, pp. 2601-2608.

[4] K. Deb, L. Zhu, and S. Kulkarni, "Handling multiple scenarios in evolutionary multiobjective numerical optimization," IEEE Transactions on Evolutionary Computation, vol. 22, no. 6, pp. 920-933, 2018.

[5] H. Wang, J. Doherty, and Y. Jin, "Hierarchical surrogate-assisted evolutionary multi-scenario airfoil shape optimization," in 2018 IEEE Congress on Evolutionary Computation (CEC). IEEE, 2018, pp. 1-8.

[6] A. Ben-Tal and A. Nemirovski, "Robust optimization-methodology and applications," Mathematical Programming, vol. 92, no. 3, pp. 453-480, 2002.

[7] J. W. Herrmann, "A genetic algorithm for minimax optimization problems," in Evolutionary Computation, 1999. CEC 99. Proceedings of the 1999 Congress on, vol. 2. IEEE, 1999, pp. 1099-1103.

[8] M. Á. Sainz, P. Herrero, J. Armengol, and J. Vehí, "Continuous minimax optimization using modal intervals," Journal of Mathematical Analysis and Applications, vol. 339, no. 1, pp. 18-30, 2008.

[9] M. Inuiguchi and M. Sakawa, "Minimax regret solution to linear programming problems with an interval objective function," European Journal of Operational Research, vol. 86, no. 3, pp. 526-536, 1995.

[10] B. Lu, Y. Cao, M. jie Yuan, and J. Zhou, "Reference variable methods of solving min-max optimization problems," Journal of Global Optimization, vol. 42, no. 1, pp. 1-21, 2008.

[11] Y. Feng, L. Hongwei, Z. Shuisheng, and L. Sanyang, "A smoothing trustregion newton-cg method for minimax problem," Applied Mathematics and Computation, vol. 199, no. 2, pp. 581-589, 2008.

[12] H. Aissi, C. Bazgan, and D. Vanderpooten, "Min-max and min-max regret versions of combinatorial optimization problems: A survey," European journal of operational research, vol. 197, no. 2, pp. 427-438, 2009.

[13] R. Montemanni, L. M. Gambardella, and A. V. Donati, "A branch and bound algorithm for the robust shortest path problem with interval data," Operations Research Letters, vol. 32, no. 3, pp. 225-232, 2004.

[14] P. Kouvelis and G. Yu, Robust discrete optimization and its applications. Springer Science \& Business Media, 2013, vol. 14.

[15] R. Montemanni and L. M. Gambardella, "A branch and bound algorithm for the robust spanning tree problem with interval data," European Journal of Operational Research, vol. 161, no. 3, pp. 771-779, 2005.

[16] M.-J. Tahk and B.-C. Sun, "Coevolutionary augmented lagrangian methods for constrained optimization," IEEE Transactions on Evolutionary Computation, vol. 4, no. 2, pp. 114-124, 2000.

[17] A. Zhou and Q. Zhang, "A surrogate-assisted evolutionary algorithm for minimax optimization," in Evolutionary Computation (CEC), 2010 IEEE Congress on. IEEE, 2010, pp. 1-7.

[18] J. Branke and J. Rosenbusch, "New approaches to coevolutionary worstcase optimization," in International Conference on Parallel Problem Solving from Nature. Springer, 2008, pp. 144-153.

[19] X. Qiu, J.-X. Xu, Y. Xu, and K. C. Tan, "A new differential evolution algorithm for minimax optimization in robust design," IEEE transactions on cybernetics, vol. 48, no. 5, pp. 1355-1368, 2018.

[20] L. Jiao, H. Wang, R. Shang, and F. Liu, "A co-evolutionary multiobjective optimization algorithm based on direction vectors," Information Sciences, vol. 228, pp. 90-112, 2013.

[21] Y. Shi and R. A. Krohling, "Co-evolutionary particle swarm optimization to solve min-max problems," in Evolutionary Computation, 2002. CEC'02. Proceedings of the 2002 Congress on, vol. 2. IEEE, 2002, pp. $1682-1687$.

[22] R. A. Krohling, F. Hoffmann, and L. S. Coelho, "Co-evolutionary particle swarm optimization for min-max problems using gaussian distribution," in Evolutionary Computation, 2004. CEC2004. Congress on, vol. 1. IEEE, 2004, pp. 959-964.

[23] H. J. Barbosa, "A coevolutionary genetic algorithm for constrained optimization," in Evolutionary Computation, 1999. CEC 99. Proceedings of the 1999 Congress on, vol. 3. IEEE, 1999, pp. 1605-1611.

[24] M. T. Jensen, "A new look at solving minimax problems with coevolutionary genetic algorithms," in Metaheuristics: computer decisionmaking. Springer, 2003, pp. 369-384.

[25] A. M. Cramer, S. D. Sudhoff, and E. L. Zivi, "Evolutionary algorithms for minimax problems in robust design," IEEE Transactions on Evolutionary Computation, vol. 13, no. 2, pp. 444-453, 2009. 
[26] E. Laskari, K. Parsopoulos, and M. Vrahatis, "Particle swarm optimization for minimax problems," in Evolutionary Computation, 2002. CEC'02. Proceedings of the 2002 Congress on, vol. 2. IEEE, 2002, pp. $1576-1581$.

[27] B. Liu, Q. Zhang, and G. G. Gielen, "A Gaussian process surrogate model assisted evolutionary algorithm for medium scale expensive optimization problems," IEEE Transactions on Evolutionary Computation, vol. 18, no. 2, pp. 180-192, 2014.

[28] Y. Jin, "A comprehensive survey of fitness approximation in evolutionary computation," Soft Computing, vol. 9, no. 1, pp. 3-12, 2005.

[29] — "Surrogate-assisted evolutionary computation: Recent advances and future challenges," Swarm and Evolutionary Computation, vol. 1, no. 2, pp. 61-70, 2011.

[30] Y. Jin, H. Wang, T. Chugh, D. Guo, and K. Miettinen, "Data-driven evolutionary optimization: An overview and case studies," IEEE Transactions on Evolutionary Computation, 2018.

[31] Z. Zhou, Y. S. Ong, P. B. Nair, A. J. Keane, and K. Y. Lum, "Combining global and local surrogate models to accelerate evolutionary optimization," IEEE Transactions on Systems, Man, and Cybernetics, Part C: Applications and Reviews, vol. 37, no. 1, pp. 66-76, 2007.

[32] D. Lim, Y. Jin, Y.-S. Ong, and B. Sendhoff, "Generalizing surrogateassisted evolutionary computation," IEEE Transactions on Evolutionary Computation, vol. 14, no. 3, pp. 329-355, 2010.

[33] H. Wang, Y. Jin, and J. Doherty, "Committee-based active learning for surrogate-assisted particle swarm optimization of expensive problems," IEEE Transactions on Cybernetics, vol. 47, no. 9, pp. 2664-2677, 2017.

[34] D. Buche, N. N. Schraudolph, and P. Koumoutsakos, "Accelerating evolutionary algorithms with gaussian process fitness function models," IEEE Transactions on Systems, Man, and Cybernetics, Part C: Applications and Reviews, vol. 35, no. 2, pp. 183-194, 2005.

[35] T. Chugh, Y. Jin, K. Miettinen, J. Hakanen, and K. Sindhya, "A surrogate-assisted reference vector guided evolutionary algorithm for computationally expensive many-objective optimization," IEEE Transactions on Evolutionary Computation, vol. 22, no. 1, pp. 129 - 142, 2018.

[36] J. Tian, Y. Tan, J. Zeng, C. Sun, and Y. Jin, "Multi-objective infill criterion driven gaussian process assisted particle swarm optimization of high-dimensional expensive problems," IEEE Transactions on Evolutionary Computation, 2018.

[37] C. Sun, Y. Jin, R. Cheng, J. Ding, and J. Zeng, "Surrogate-assisted cooperative swarm optimization of high-dimensional expensive problems," IEEE Transactions on Evolutionary Computation, vol. 21, no. 4, pp. 644-660, 2017.

[38] D. Guo, Y. Jin, J. Ding, and T. Chai, "Heterogeneous ensemble-based infill criterion for evolutionary multiobjective optimization of expensive problems," IEEE Transactions on Cybernetics, 2018.

[39] H. Wang, Y. Jin, C. Sun, and J. Doherty, "Offline data-driven evolutionary optimization using selective surrogate ensembles," IEEE Transactions on Evolutionary Computation, 2018.

[40] Y.-S. Ong, P. B. Nair, and K. Lum, "Max-min surrogate-assisted evolutionary algorithm for robust design," IEEE Transactions on Evolutionary Computation, vol. 10, no. 4, pp. 392-404, 2006.

[41] Y.-S. Ong and A. Gupta, "Evolutionary multitasking: a computer science view of cognitive multitasking," Cognitive Computation, vol. 8, no. 2 , pp. 125-142, 2016

[42] A. Gupta, Y.-S. Ong, and L. Feng, "Multifactorial evolution: toward evolutionary multitasking," IEEE Transactions on Evolutionary Computation, vol. 20, no. 3, pp. 343-357, 2016.

[43] — - "Insights on transfer optimization: Because experience is the best teacher," IEEE Transactions on Emerging Topics in Computational Intelligence, vol. 2, no. 1, pp. 51-64, 2018.

[44] A. Gupta and Y.-S. Ong, "Genetic transfer or population diversification? deciphering the secret ingredients of evolutionary multitask optimization," in 2016 IEEE Symposium Series on Computational Intelligence (SSCI). IEEE, 2016, pp. 1-7.

[45] A. Gupta, Y.-S. Ong, L. Feng, and K. C. Tan, "Multiobjective multifactorial optimization in evolutionary multitasking," IEEE transactions on cybernetics, vol. 47, no. 7, pp. 1652-1665, 2017.

[46] C. Yang, J. Ding, Y. Jin, C. Wang, and T. Chai, "Multitasking multiobjective evolutionary operational indices optimization of beneficiation processes," IEEE Transactions on Automation Science and Engineering, no. 99 , pp. 1-12, 2018.

[47] N. Q. Tuan, T. D. Hoang, and H. T. T. Binh, "A guided differential evolutionary multi-tasking with powell search method for solving multiobjective continuous optimization," in 2018 IEEE Congress on Evolutionary Computation (CEC). IEEE, 2018, pp. 1-8.
[48] L. Feng, L. Zhou, J. Zhong, A. Gupta, Y.-S. Ong, K.-C. Tan, and A. Qin, "Evolutionary multitasking via explicit autoencoding," IEEE transactions on cybernetics, no. 99, pp. 1-14, 2018.

[49] A. Gupta, J. Mańdziuk, and Y.-S. Ong, "Evolutionary multitasking in bi-level optimization," Complex \& Intelligent Systems, vol. 1, no. 1-4, pp. 83-95, 2015.

[50] J. Ding, C. Yang, Y. Jin, and T. Chai, "Generalized multi-tasking for evolutionary optimization of expensive problems," IEEE Transactions on Evolutionary Computation, 2017.

[51] J. Zhong, L. Feng, W. Cai, and Y.-S. Ong, "Multifactorial genetic programming for symbolic regression problems," IEEE Transactions on Systems, Man, and Cybernetics: Systems, no. 99, pp. 1-14, 2018.

[52] H. Li, Y.-S. Ong, M. Gong, and Z. Wang, "Evolutionary multitasking sparse reconstruction: Framework and case study," IEEE Transactions on Evolutionary Computation, 2018.

[53] L. Zhou, L. Feng, J. Zhong, Y.-S. Ong, Z. Zhu, and E. Sha, "Evolutionary multitasking in combinatorial search spaces: A case study in capacitated vehicle routing problem," in 2016 IEEE Symposium Series on Computational Intelligence (SSCI). IEEE, 2016, pp. 1-8.

[54] S. Tsutsui and A. Ghosh, "Genetic algorithms with a robust solution searching scheme," IEEE transactions on Evolutionary Computation, vol. 1, no. 3, pp. 201-208, 1997

[55] H. Wang, Q. Zhang, L. Jiao, and X. Yao, "Regularity model for noisy multiobjective optimization," IEEE transactions on cybernetics, vol. 46, no. 9, pp. 1997-2009, 2015.

[56] C. R. Cloninger, J. Rice, and T. Reich, "Multifactorial inheritance with cultural transmission and assortative mating. ii. a general model of combined polygenic and cultural inheritance." American journal of human genetics, vol. 31, no. 2, p. 176, 1979.

[57] K.-L. Du and M. Swamy, "Radial basis function networks," in Neural Networks and Statistical Learning. Springer, 2014, pp. 299-335.

[58] J. Derrac, S. García, D. Molina, and F. Herrera, "A practical tutorial on the use of nonparametric statistical tests as a methodology for comparing evolutionary and swarm intelligence algorithms," Swarm and Evolutionary Computation, vol. 1, no. 1, pp. 3-18, 2011.

[59] J. J. Doherty, H. Wang, and Y. Jin, "Use of global drag rise boundaries to investigate ill-posed transonic airfoil optimization," in 2018 Applied Aerodynamics Conference, 2018, p. 3953.

[60] R. M. Hicks and P. A. Henne, "Wing design by numerical optimization," Journal of Aircraft, vol. 15, no. 7, pp. 407-412, 1978.

[61] J. Zhang, W. Zhou, X. Chen, W. Yao, and L. Cao, "Multi-source selective transfer framework in multi-objective optimization problems," IEEE Transactions on Evolutionary Computation, pp. 1-1, 2019. 